\title{
Post-translational modifications and secretion of Wnt proteins
}

\author{
Yanqin Lu' ${ }^{1,2 *}$, Xiuzh Ren ${ }^{3}$, YanzhouWang ${ }^{4}$ and Jinxiang $\operatorname{Han}^{1,2}$ \\ Biotechnology Centre, Shandong Academy of Medical Sciences, China \\ ${ }^{2}$ School of Medicine and Life Sciences, University of Jinan-Shandong Academy of Medical Sciences, China \\ ${ }^{3}$ Department of Orthopedic Surgery, The People's Hospital of Wuqing District, China \\ ${ }^{4}$ Department of Orthopedic Surgery, Shandong Provincial Hospital, China
}

${ }^{1}$ Key Laboratory for Biotech-Drugs Ministry of Health, Key Laboratory for Rare \& Uncommon Diseases of Shandong Province, Shandong Medicinal

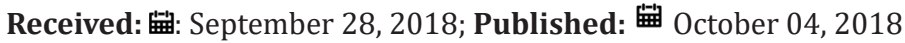

*Corresponding author: Yanqin Lu, Key Laboratory for Biotech-Drugs Ministry of Health, Key Laboratory for Rare \& Uncommon Diseases of Shandong Province, Shandong Medicinal Biotechnology Centre, Shandong Academy of Medical Sciences, China

\begin{abstract}
Wnts are secreted lipid-modified glycoproteins in most mammalian genomes. Wnt ligands are the essential components of Wnt signaling pathways. Before binding to specific receptors, Wnt proteins experienced post-translational modification of acylation and N-glycosylation mediated by acyltransferase porcupine. Mature Wnts are transported from the Golgi to plasma membrane under association with Wntless. Retromer complex, P24 and other molecules are all involved in the secretion and release of Wnts. In this review, we provide an update of the post-translational modification, secretion and release of Wnts.
\end{abstract}

Keywords: Wnts; Acylation; N glycosylation; Wntless; Retromer

\section{Introduction}

Wnt proteins are a family of cysteine-rich secreted glycoproteins of approximately 350-400 amino acids in length [1]. Averagely, Whts contains several charged residues and 23 -25 cysteines, some of them are associated with the formation of inter- and intramolecular disulfide bonds that stabilize proper Wnt folding and multimerization [2,3]. Till now, 19 mammalian Wnt proteins have been identified to fall into 12 conserved Wnt subfamilies. By binding to 10 different Frizzled receptor and other co-receptors, Wnt starts its cellular signal transduction. Wht signaling transduction is essential in embryonic development, cell proliferation, cell migration, cell fate specification, and axis patterning. In the past decades, great achievement has been made in the Wnt and Wnt signaling transduction, initial from mouse and Drosophila model study. We review the progress of post-translational modifications of Wnts in the endoplasmic reticulum (ER) and Golgi apparatus, and further secretion of Wnt proteins.

\section{Post-Translational Modifications of Wnt Proteins}

Wht proteins secreted from cells are post-translationally modified before transporting into the extracellular space. Aminoterminal signal peptide guides Wnt proteins to the ER, where Wnts undergo a series of post-transcriptional modifications in the secretory pathway. Post-translational acylation and N-glycosylation is observed in all Wnt proteins, with exception of Drosophila WntD [4]. They are independent modification and N-glycosylation precedes palmitoylation $[5,6]$. Both acylation and N-glycosylation of Wnt proteins are mediated by acyltransferase porcupine, which is localized at ER $[4,7,8]$.

Acylation modification is reported to be vital for the secretion of Wnt proteins and for Wnt binding to the co-receptor Frizzled, the chaperone Wntless (Wls) and signaling activity $[3,7,9,10]$. Initially, two conserved residues of fatty acylation are identified. One is cysteine residue 77 (murine Wnt3a), where palmitate is attached through a thioester linkage. The other was serine residue 209 (murine Wnt3a), which is modified by a monounsaturated fatty acid, palmitoleic acid $[7,11,12]$. Wnt3a mutant with serine to alanine at residue of 209 is retained in the ER and secretion is blocked [11]. XWnt8 structures support that only conserved serine 229 (murine Wnt3a) is acylated. Cys77 participate in the formation of disulfide bond with a second conserved cysteine residue [3]. Mono-acylation is further proved by the lack of Cys77 palmitoylation study $[13,14]$. Palmitoyleolation process of Wnt proteins is reversible and it can be removed by the serine hydrolase of NOTUM specifically $[15,16]$.

The role of N-glycosylation is unclear, usually, it influences secretion of Wnt proteins, but not folding and structure [4]. There 
are no conserved $\mathrm{N}$-glycosylation sites in different Wnt members [17]. Wg protein has two known N-glycosylation sites of Asn103 and Asn414, Wg mutant can also activate downstream signaling in both autocrine and paracrine signaling, though secretion ability is reduced [17]. Meanwhile, Loss of N-glycosylation of Wnt1 impairs paracrine signaling. For Wnt3a and Wnt5a, N-glycosylation is required for secretion, but not for their activities $[6,18]$.

There are also other post-translational modifications identified in Wnt proteins. Tyrosine sulfation of Wnt5a and Wnt11 is essential for the formation of Wnt5a/Wnt11 complexes, which induce the efficient signaling in the initiation of Xenopus axis formation [19]. Wnt1 are attached to glycosylphosphatidylinositol (GPI) anchor on the leaflet of the plasma membrane (PM) by the glycolipid tail. PGAP1 gene participate this modification by creating a hydrophobic Wnt1 that is retained in the ER [20].

\section{Secretion and Release of Wnt Proteins}

Mature Wnt proteins are transported from the Golgi to the PM for secretion by the conserved multi-pass transmembranse Wls receptor (GPR177 in mammals) after post-translational modification [21]. Wht secretion is Wls-dependent; it cannot proceed with the absence of Wls [22]; While other signaling proteins are not influenced by the removal of Wls [23-25]. Wls has a carboxy-terminal ER-targeting signal, which guiding Wls localizing predominantly in the ER, where it binds with acylated Wnt proteins $[9,26]$. Then, Wnts-Wls complex transports from ER to PM in the presence of COPII vesicles. Wnt is released and binds to lipoprotein particles or heparin sulpate proteoglycans (HSPGs) once Wnts-Wls complex arriving at the PM $[27,28]$. However, other theory supports that Wnts-Wls complex keep together and internalized at PM and dissociate from each other in endosomes. Then, Wnts are released through a recycling endosomal pathway and Wls is transported back to trans-Golgi network (TGN) though a retromer-dependent pathway [28-30]. Endocytosed Wls progresses to multivesicular bodies (MVBs) and lysosomes for degradation [31].

Retromer is a complex, consisting of Vps35, Vps29, Vps26, Vps10, Vps5, and Vps17 in yeast [32-34]. Vps35, Vps29 and Vps26 subcomplex serve as cargo recognition and retrieve Vps10 from endosomes to the Golgi [33]. Vps5 and Vps17 are membranebound subcomplexs, they are sorting nexins (SNX) with a phosphoinositide-binding SNX-phox homology (SNX-PX) domain [35]. Nexins SNX1, SNX2, SNX5 and SNX6 are SNX-BAR coat complex interacting with cargo-selective Vps35-Vps29-Vps26 complex. They are needed for most of the retromer cargo proteins [35,36]. SNX3 co-interact with Wls and Vps26 on early endosomes and help the association of the cargo-selective complex to Wls. Wls recycling relies on SNX3 specifically [36,37]. Wls recycled in Golgi is further retrograde transport to ER in a COPI- dependent way and require ER-Golgi intermediate compartment ERGIC2 [26,38].

The mechanisms of Wnt secretion and release are complex, more and more molecules are discovered to be related with them. P24 protein family, which acts as cargo receptor for Wnt in the early secretory pathway, is important for proper export of $\mathrm{Wg}$ from the ER
[39-41]. Sec22 is associated with both Wg and p24 during the early secretory phase of Wg [41]. MiR-307a inhibits Wg secretion through targeting Wls, its overexpression induces ER stress specifically in the Wg-expressing cells [38]. Wnts are classic morphogens. They are vital for tissue patterning by activating their target genes in a concentration-dependent manner $[13,42]$. Secreted Wnts associate to specific receptors on target cells to activate canonical Wnt/ $\beta$ catenin pathway, or Wnt/Ca2+, Wnt/ planar cell polarity and other non-canonical pathways.

\section{Acknowledgement}

The work was supported by Grants-in-Aid from Shandong government (No. 2016ZDJS07A10, No.2016GSF201222), the State Major Infectious Disease Research (China Central Government, 2017ZX10103004-007).

\section{References}

1. Kusserow A, Pang K, Sturm C, Hrouda M, Lentfer J, et al. (2005) Unexpected complexity of the Wnt gene family in a sea anemone. Nature 433(7022): 156-160.

2. Tanaka K, Kitagawa Y, Kadowaki T (2002) Drosophila segment polarity gene product porcupine stimulates the posttranslational N-glycosylation of wingless in the endoplasmic reticulum. The Journal of biological chemistry 277(15): 12816-12823.

3. Janda CY, Waghray D, Levin AM, Thomas C, Garcia KC (2012) Structural basis of Wnt recognition by Frizzled. Science 337(6090): 59-64.

4. Coudreuse D, Korswagen HC (2007) The making of Wnt: New insights into Wnt maturation, sorting and secretion. Development 134(1): 3-12.

5. Komekado H, Yamamoto H, Chiba T, Kikuchi A (2007) Glycosylation and palmitoylation of Wnt-3a are coupled to produce an active form of Wnt3a. Genes to cells: Devoted to molecular \& cellular mechanisms 12(4): 521-534.

6. Doubravska L, Krausova M, Gradl D, Vojtechova M, Tumova L, et al. (2011) Fatty acid modification of Wnt1 and Wnt3a at serine is prerequisite for lipidation at cysteine and is essential for Wnt signalling. Cellular signalling 23(5): 837-848.

7. Willert K, Brown JD, Danenberg E, Duncan AW, Weissman IL, et al. (2003) Wnt proteins are lipid-modified and can act as stem cell growth factors. Nature 423(6938): 448-452.

8. Ching W, Nusse R (2006) A dedicated Wnt secretion factor. Cell 125(3): 432-433.

9. Coombs GS, Yu J, Canning CA, Veltri CA, Covey TM, et al. (2010) WLSdependent secretion of WNT3A requires Ser209 acylation and vacuolar acidification. Journal of cell science 123(19): 3357-3367.

10. Herr P, Basler K (2012) Porcupine-mediated lipidation is required for Wnt recognition by Wls. Developmental biology 361(2): 392-402.

11. Takada R, Satomi Y, Kurata T, Ueno N, Norioka S, et al. (2006) Monounsaturated fatty acid modification of Wnt protein: its role in Wnt secretion. Developmental cell 11(6): 791-801.

12. Franch Marro X, Wendler F, Griffith J, Maurice MM, Vincent JP (2008) In vivo role of lipid adducts on Wingless. Journal of cell science 121(10): 1587-1592.

13. Parchure A, Vyas N, Mayor S (2018) Wnt and Hedgehog: Secretion of Lipid-Modified Morphogens. Trends in cell biology 28(2): 157-170.

14. Rios-Esteves J, Haugen B, Resh (2014) Identification of key residues and regions important for porcupine-mediated Wnt acylation. The Journal of biological chemistry 289(24): 17009-17019. 
15. De Gregorio R, Pulcrano S, De Sanctis C, Volpicelli F, Guatteo E, et al. (2018) miR-34b/c Regulates Wnt1 and Enhances Mesencephalic Dopaminergic Neuron Differentiation. Stem cell reports 10(4): 12371250.

16. Gross JC, Zelarayan LC (2018) The Mingle-Mangle of Wnt Signaling and Extracellular Vesicles: Functional Implications for Heart Research. Frontiers in cardiovascular medicine 5: p.10.

17. Tang X, Wu Y, Belenkaya TY, Huang Q Ray L, et al. (2012) Roles of $\mathrm{N}$-glycosylation and lipidation in $\mathrm{Wg}$ secretion and signaling. Developmental biology 364(1): 32-41.

18. Kurayoshi M, Yamamoto H, Izumi S, Kikuchi A (2007) Post-translational palmitoylation and glycosylation of Wnt-5a are necessary for its signalling. The Biochemical journal 402(3): 515-523.

19. Cha SW, Tadjuidje E, White J, Wells J, Mayhew C, et al. (2009) Wnt11/5a complex formation caused by tyrosine sulfation increases canonical signaling activity. Current biology: CB 19(18): 1573-1580.

20. Kinoshita T, Maeda Y, Fujita M (2013) Transport of glycosylphosphatidylinositol-anchored proteins from the endoplasmic reticulum. Biochimica et biophysica acta 1833(11): 2473-2478.

21. Das S, Yu S, Sakamori R, Stypulkowski E, Gao N (2012) Wntless in Wnt secretion: Molecular, cellular and genetic aspects. Frontiers in biology 7(6): 587-593.

22. Fu J, Jiang M, Mirando AJ, Yu HM, Hsu W (2009) Reciprocal regulation of Wnt and Gpr177/mouse Wntless is required for embryonic axis formation. Proceedings of the National Academy of Sciences of the United States of America 106(44): 18598-18603.

23. Langton PF, Kakugawa S, Vincent JP (2016) Making, Exporting, and Modulating Wnts. Trends in cell biology 26(10): 756-765.

24. Banziger C, Soldini D, Schutt C, Zipperlen P, Hausmann G, et al. (2006) Wntless, a conserved membrane protein dedicated to the secretion of Wnt proteins from signaling cells. Cell 125(3): 509-522.

25. Bartscherer K, Pelte N, Ingelfinger D, Boutros M (2006) Secretion of Wnt ligands requires Evi, a conserved transmembrane protein. Cell 125(3): 523-533.

26. Yu J, Chia J, Canning CA, Jones CM, Bard FA, et al. (2014) WLS retrograde transport to the endoplasmic reticulum during Wht secretion. Developmental cell 29(3): 277-291.

27. Panakova D, Sprong H, Marois E, Thiele C, Eaton S (2005) Lipoprotein particles are required for Hedgehog and Wingless signalling. Nature 435(7038): 58-65.

28. Lorenowicz MJ, Korswagen HC (2009) Sailing with the Wnt: Charting the Wnt processing and secretion route. Experimental cell research 315(16): 2683-2689.

ISSN: 2574-1241

DOI: 10.26717/BJSTR.2018.09.001824

Yanqin Lu. Biomed J Sci \& Tech Res

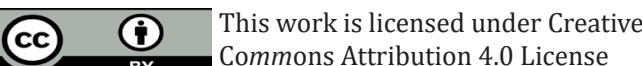

Submission Link: https://biomedres.us/submit-manuscript.php
29. Port F, Kuster M, Herr P, Furger E, Banziger C, et al. (2008) Wingless secretion promotes and requires retromer-dependent cycling of Wntless. Nature cell biology 10(2): 178-185.

30. Yang PT, Lorenowicz MJ, Silhankova M, Coudreuse DY, Betist MC, et al. (2008) Wnt signaling requires retromer-dependent recycling of MIG14/Wntless in Wnt-producing cells. Developmental cell 14(1): 140-147.

31. Seaman MN (2012) The retromer complex - endosomal protein recycling and beyond. Journal of cell science 125: 4693-4702.

32. Hausmann G, Banziger C, Basler K (2007) Helping Wingless take flight: How WNT proteins are secreted. Nature reviews Molecular cell biology 8(4): 331-336.

33. Seaman MN (2005) Recycle your receptors with retromer. Trends in cell biology 15(2): 68-75.

34. Eaton S (2008) Retromer retrieves wntless. Developmental cell 14(1): 4-6.

35. Cullen PJ (2008) Endosomal sorting and signalling: An emerging role for sorting nexins. Nature reviews Molecular cell biology 9(7): 574-582.

36. Harterink M, Port F, Lorenowicz MJ, McGough IJ, Silhankova M, et al. (2011) A SNX3-dependent retromer pathway mediates retrograde transport of the Wnt sorting receptor Wntless and is required for Wnt secretion. Nature cell biology 13(8): 914-923.

37. Johannes L, Wunder C (2011) The SNXy flavours of endosomal sorting. Nature cell biology 13(8): 884-886.

38. Zhang P, Zhou L, Pei C, Lin X, Yuan Z (2016) Dysfunction of Wntless triggers the retrograde Golgi-to-ER transport of Wingless and induces ER stress. Scientific reports 6: 19418.

39. Buechling T, Chaudhary V, Spirohn K, Weiss M, Boutros M (2011) p24 proteins are required for secretion of Wnt ligands. EMBO reports 12(12): 1265-1272.

40. Port F, Hausmann G, Basler K (2011) A genome-wide RNA interference screen uncovers two p24 proteins as regulators of Wingless secretion. EMBO reports 12(11): 1144-1152.

41. Li X, Wu Y, Shen C, Belenkaya TY, Ray L, et al. (2015) Drosophila p24 and Sec22 regulate Wingless trafficking in the early secretory pathway. Biochemical and biophysical research communications 463(4): 483489.

42. Ashe HL, Briscoe J (2006) The interpretation of morphogen gradients. Development 133(3): 385-394.

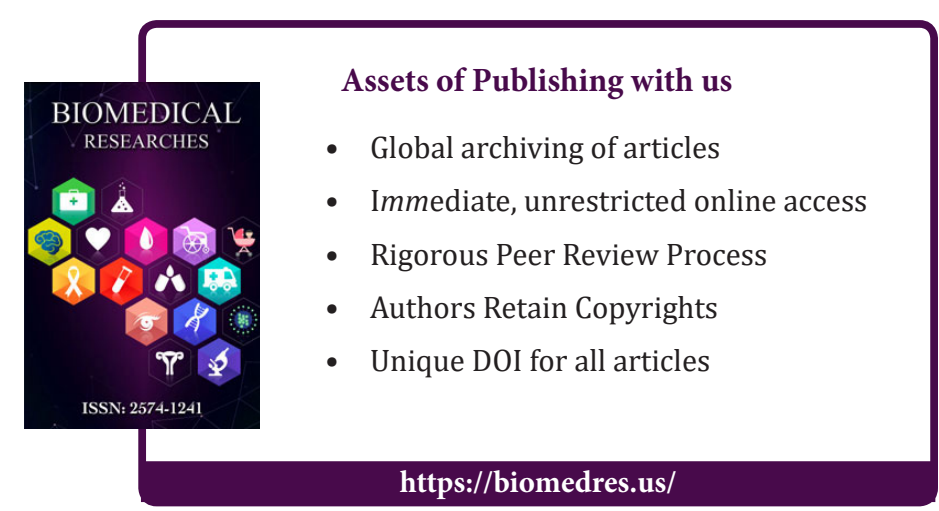

Cite this article: Yanqin L, Xiuzh R, Yanzhou W, Jinxiang H. Post-translational modifications and secretion of Wnt proteins. Biomed J Sci\&Tech

Res 9(4)-2018. BJSTR. MS.ID.001824. DOI: 10.26717/ BJSTR.2018.09.001824. 